\title{
Correlación histológica de lesiones ultrasonográficas de mama mediante una biopsia con aguja de corte grueso asistida por succión
}

\section{Histological correlation in ultrasonography breast features by suction biopsy with thick-cut needle assisted}

\author{
Ludwig Isaac Arruel Caraveo, ${ }^{*}$ Víctor Manuel Marroquín Torres, ${ }^{\neq}$ \\ Eric Misael Saucedo Moreno, § Mariana Torre Inclán, "| María Pilar Rodríguez Reséndiz
}

\section{Resumen}

Introducción: En México, el cáncer de mama se diagnostica en promedio a los 53 años de edad, lo que representa casi una década menor en comparación con otros países. Estudios clínicos han demostrado que el tamizaje con mastografía reduce $24 \%$ la mortalidad por cáncer de mama. Material y métodos: Se realizó un estudio retrospectivo de concordancia entre mastografía diagnóstica y biopsia con aguja de corte gruesa asistida por succión. Resultados: Se analizaron 870 casos entre 2005 y 2015; todos los pacientes evaluados tenían reporte por ultrasonido de BIRADS (Breast Imaging Report and Database System) 4 o 5, o bien, sospecha clínica, fobia a cáncer o deseo de la paciente. Se demostró una sensibilidad de 0.99 , especificidad de 0.24 , valor predictivo positivo de 0.28 , valor predictivo negativo de 0.98 y exactitud de 0.42 , siendo mucho más precisos cuando se trataban de lesiones BIRADS 4C y 5, cuyos valores fueron significativamente mejores. Conclusiones: Los resultados reportados en este estudio traducen que la mastografía diagnóstica tiene una alta correlación con el resultado histopatológico.

Palabras clave: Cáncer de mama, tumor mamario, ultrasonido mamario.

\section{Abstract}

Introduction: In México, breast cancer is diagnosed on average at 53 years of age, which represents almost a decade less compared to other countries. Clinical studies have shown that mammography screening reduces breast cancer mortality by $24 \%$. Material and methods: A retrospective study of concordance between diagnostic mammography and biopsy with thick-cut needle assisted by suction was performed. Results: 870 cases were analyzed between 2005 and 2015; all patients evaluated had a BIRADS (Breast Imaging Report and Database System) 4 or 5 report, or clinical suspicion, cancer phobia or desire of the patient. We observed a sensivity 0.99 , specificity 0.24 , PPV 0.28 , NPV 0.98 and accuracy 0.42 , being much more accurate when dealing whit BIRADS $4 \mathrm{C}$ and 5 lesions, whose values were significantly better. Conclusions: The results that reported in this study show that diagnostic mammography has a high correlation with the histopathological result.

Keywords: Breast cancer, breast tumor, breast ultrasound.
* Cirujano General. Facultad Mexicana de Medicina de la Universidad La Salle México. Servicio de Cirugía Oncológica, Hospital Ángeles Mocel, CMDX, México.

* Cirujano Oncólogo. Departamento de Oncología Quirúrgica. Hospital Ángeles Mocel, CDMX, México.

$\S$ Cirujano General y Asesor Estadístico. Servicio de Cirugía General, Hospital Ángeles Mocel, CDMX, México.

I Residente de Ginecología y Obstetricia. UNAM, Hospital Español, CDMX, México.
" Médico General, Servicio de Cirugía Oncología. Hospital Ángeles Mocel, CDMX, México.

Correspondencia:

Dr. Ludwig Isaac Arruel Caraveo

Correo electrónico: oncomed1@hotmail.com

Aceptado: 01-11-2019. 


\section{INTRODUCCIÓN}

El cáncer de mama es el cáncer más frecuente en las mujeres. Se estima que tiene una incidencia de alrededor de 690 mil nuevos casos por año (a razón de uno por cada cuatro habitantes). ${ }^{1}$ Asimismo, es la primera causa de muerte por neoplasia en la mujer, con cerca de 500 mil decesos cada año, y de éstos, $70 \%$ ocurre en países en desarrollo. ${ }^{2}$

Los programas de tamizaje buscan disminuir la mortalidad de una población determinada y al mismo tiempo, aunque no es su objetivo principal, reducir los costos de atención.

En México, el primer programa de pesquisa con mastografía se reportó en el 2009. ${ }^{3}$ Éste hace énfasis en el porcentaje de casos reportados como el BIRADS 0, 4 y 5 (Breast Imaging Report and Database System), que fue de $1 \%$ en $2005-2006$ a $3.44 \%$ en 2007 y que requiere estudios subsecuentes, biopsias y/o tratamiento. El porcentaje más bajo fue reportado en series europeas o americanas con cerca de 2 a 8\%. Esta diferencia puede indicar que nuestra población efectivamente tiene menor frecuencia de lesiones malignas, o bien que estamos diagnosticando menos casos sospechosos.

Es de suma importancia conocer adecuadamente la confiabilidad de los estudios de pesquisa que se realizan en cada unidad, ya que ésta depende de múltiples factores directamente asociados con el lugar (incluyendo recurso humano) donde se realizan. La variabilidad de los estudios es sustancial, y no es posible tomar los datos de otros países, porque los sistemas de salud son diferentes y todas las variables de las cuales depende la confiabilidad de los estudios de imagen en mama también son diferentes. Es por ello que consideramos imprescindible realizar este estudio, con el fin de conocer mejor la confiabilidad de la prueba dentro y fuera de nuestra unidad.

\section{MATERIAL Y MÉTODOS}

Se realizó un estudio retrospectivo, transversal, observacional y analítico. Éste consistió en la revisión de expedientes

Tabla 1: Número de casos con base en la cantidad de focos detectados con sospecha.

$\begin{array}{ccc}\text { Núm. de focos } & \text { Unilateral n (\%) } & \text { Bilateral } n(\%) \\ 1 & 741(88.4) & --- \\ 2 & 84(10.0) & 30(96.8) \\ 3 & 12(1.4) & 1(3.2) \\ 4 & 2(0.2) & --- \\ \text { Total por grupo } & 839(100) & 31(100)\end{array}$

Tabla 2: Proporciones de los diagnósticos clínicos.

\begin{tabular}{lrr} 
Hallazgo radiológico & $\mathrm{n}$ & $\%$ \\
\hline Masa & 765 & 87.9 \\
Quiste simple & 7 & 0.8 \\
Quiste complejo & 6 & 0.7 \\
Calcificaciones & 76 & 8.7 \\
Masa y calcificaciones & 13 & 1.5 \\
Masa y quiste & 3 & 0.3 \\
Total & 870 & 100.0 \\
\end{tabular}

clínicos de todas las pacientes sometidas a biopsia de mama con aguja de corte grueso asistida por succión (VACORA) y guiada por ultrasonido, durante el periodo de enero de 2005 a diciembre de 2015. Los expedientes debían cumplir con los reportes radiológicos de mama con BIRADS 4A, 4B, 4C, y 5; también, se incluyeron a todos aquellos casos BIRADS 2 y 3 con fobia, deseo de la paciente o duda diagnóstica por el oncólogo. Las pacientes contaron con estudio de imagen y reporte completo del mismo.

Se realizó una correlación con el estudio histopatológico, el cual se incluyó en una variable dicotómica expresada como benigna y maligna. Se excluyeron a aquellas pacientes en las que no se encontraron datos completos, ya fueran clínicos, de imagen o histopatológico.

Respecto al resumen de todos los reportes de patología, dado que sólo se trató de revisión de expedientes y se guardó la privacidad de las pacientes, no fue necesaria la inclusión del consentimiento informado para efectos del presente trabajo. Es importante mencionar que, con base en la normativa operacional vigente de los sistemas de Salud, todas las pacientes firmaron el consentimiento informado de manera previa a la realización de la biopsia.

Se realizó un análisis de Kolmogórov-Smirnov para determinar la distribución de las variables cuantitativas; las variables con distribuciones no paramétricas fueron analizadas con la prueba $U$ de Mann-Whitney, y se presentan en el estudio como medianas y mínimos máximos. La prueba $\chi^{2}$ o Fisher se utilizó para datos categóricos. Se consideró un valor menor a 0.05 para una $\mathrm{p}$ significativa con IC de $95 \%$. Se calculó sensibilidad, especificidad, VPN y VPP. Las curvas COR fueron utilizadas para medir la discriminación del test y los datos se analizaron utilizando el software SPSS (versión 20.0 para Windows).

\section{RESULTADOS}

Para este estudio, se incluyeron 870 pacientes con una mediana de edad de 48 años (rango 15 a 92 años). De éstos, 
Tabla 3: Concordancia diagnóstica hecha con base en el reporte BIRADS y el reporte patológico final.

\begin{tabular}{ccccc}
\hline Diagnóstico por imagen (BIRADS) & Benigno & Maligno & Total (\%) & Porcentaje por grupo \\
\hline 2 & 12 & 0 & $12(1.4)$ & 0.0 \\
3 & 153 & 2 & $155(17.8)$ & 1.2 \\
$4 \mathrm{~A}$ & 425 & 61 & $486(55.9)$ & 12.5 \\
$4 \mathrm{~B}$ & 44 & 18 & $62(7.1)$ & 29.0 \\
$4 \mathrm{C}$ & 16 & 34 & $50(5.7)$ & 88.0 \\
5 & 15 & 90 & $105(12.1)$ & 85.0 \\
Total & 665 & 205 & $870(100.0)$ & 23.0 \\
\hline BIRADS = Breast Imaging Report and Database System. & & &
\end{tabular}

839 pacientes (96.4\%) presentaron lesión unilateral y 31 casos (3.6\%) presentaron lesiones bilaterales. Setecientos cuarenta y uno (88.4\%) presentaron lesión única, 114 casos (13.1\%) dos lesiones, 13 (1.5\%) tres lesiones y dos casos $(0.2 \%)$ cuatro lesiones (Tabla 1). Los hallazgos radiológicos más frecuentes fueron: masa en 765 (87.9\%) casos, calcificaciones en 76 (8.7\%) casos; algunos otros como quistes simples, complejos, masa con calcificaciones y masa con quistes ocurrieron en menos de $2 \%$ de los casos (Tabla 2).

De acuerdo con el sistema de clasificación BIRADS, observamos una proporción de casos para BIRADS 5 de 105 (12.1\%) pacientes, 4A de 486 (55.9\%), 4B de 62 (7.1\%) y $4 \mathrm{C}$ de 50 (5.7\%). Los resultados histopatológicos fueron 665 casos (76.5\%) benignos y 205 (23.5\%) malignos. Al comparar los resultados con la clasificación BIRADS, todos los casos clasificados como BIRADS 2 fueron benignos, dos casos BIRADS 3 (1.2 \%) fueron malignos y $12.5 \%$ de los BIRADS 4A fueron malignos; para los grupos 4B, 4C y 5 fueron 29, 68 y 85\%, respectivamente (Tabla 3 y Figura 1).

Los valores representativos de la prueba diagnóstica presentaron una sensibilidad de 0.99, especificidad de 0.91, valor predictivo positivo de 0.85 y negativo de 0.98 y una exactitud de 0.42 (Tabla 4 y Figura 2).

\section{DISCUSIÓN}

El cáncer de mama se ha convertido en un problema de salud sumamente serio. El aumento exponencial de los casos hace que el sistema de salud requiera una mayor atención. Uno de los programas más importantes de tamizaje en México es el que lleva a cabo FUCAM, cuyos resultados han demostrado la detección oportuna de casos, lo cual claramente repercute en la supervivencia y en la disminución de los gastos hechos por las instituciones de salud.

El presente trabajo no evalúa directamente un sistema de tamizaje, sino la correlación entre el reporte radiológico del sistema BIRADS por ultrasonido y el reporte de patología obtenido por un método diagnóstico de elección, el cual corresponde a la biopsia con aguja de corte grueso asistido por succión y guiado por ultrasonido.

El sistema BIRADS ha sido desarrollado por el Colegio Americano de Radiología (ACR, por sus siglas en inglés); éste clasifica la densidad de la mama para reducir la discordancia en la interpretación de los hallazgos mastográficos que permita estandarizar el reporte y facilitar el seguimiento. ${ }^{4,5}$ Así mismo se atiende a la interpretación del ultrasonido y la resonancia magnética. Aún no se ha demostrado que la adición de ultrasonido a la detección mastográfica reduzca la mortalidad por cáncer de mama. ${ }^{6}$ No se han realizado ensayos aleatorios que comparen el cribado con ultrasonido suplementario y mastografía y el cribado con mastografía sola en mujeres de riesgo promedio.?

Figura 1: Proporción con base en el reporte mastográfico (BIRADS).

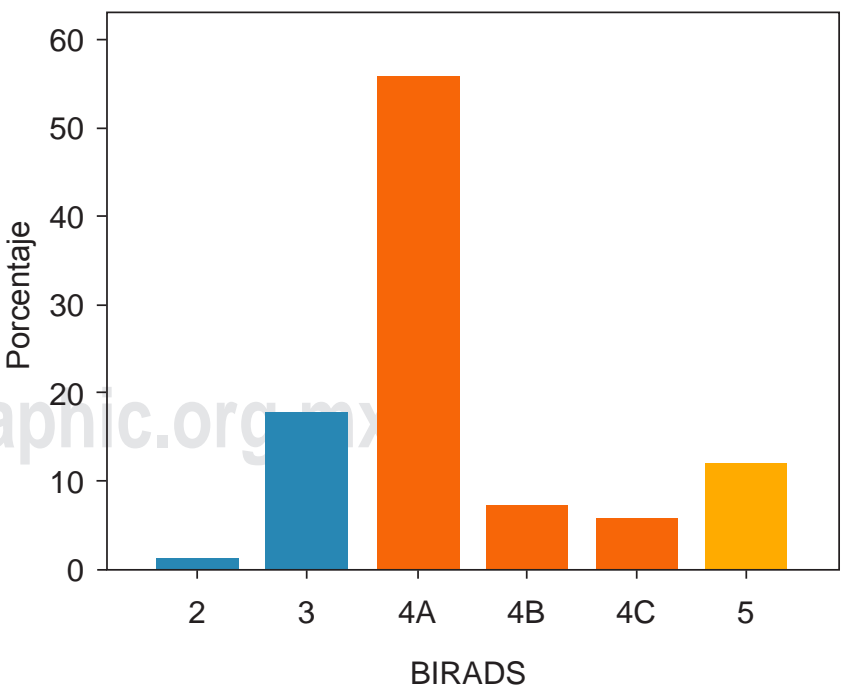

BIRADS = Breast Imaging Report and Database System . 
Tabla 4: Valores diagnósticos del estudio realizados con base en la clasificación BIRADS.

Diagnóstico por imagen

(BIRADS)
Sensibilidad

0.96

0.90

0.94

0.97
Valor predictivo

positivo
Valor predictivo negativo

0.98

0.98

0.98

0.98
Exactitud

0.34

0.79

0.91

0.93

BIRADS = Breast Imaging Report and Database System.

A manera de resumen, el BIRADS 1 representa un estudio negativo y BIRADS 2 hace referencia a hallazgos benignos; BIRADS 3 a hallazgos probablemente benignos con sospecha de malignidad menor al dos por ciento que amerita un seguimiento estrecho, por lo menos cada seis meses y BIRADS 4, a aquéllos con sospecha de malignidad. Este último se subclasifica en BIRADS 4A con un porcentaje de malignidad de $2-9 \%$, 4B con una probabilidad de malignidad de $10-49 \%$ y $4 \mathrm{C}$ probabilidad de malignidad de 50-94\%. El BIRADS 5 hace referencia a la alta sospecha de malignidad entre $95-100 \%$ y BIRADS 6 a la malignidad comprobada por biopsia.

Todos los reportes BIRADS 4 o 5 ameritan una intervención. En la gran mayoría de las instituciones, se realiza una

Figura 2: Curva ROC BIRADS vs histología.

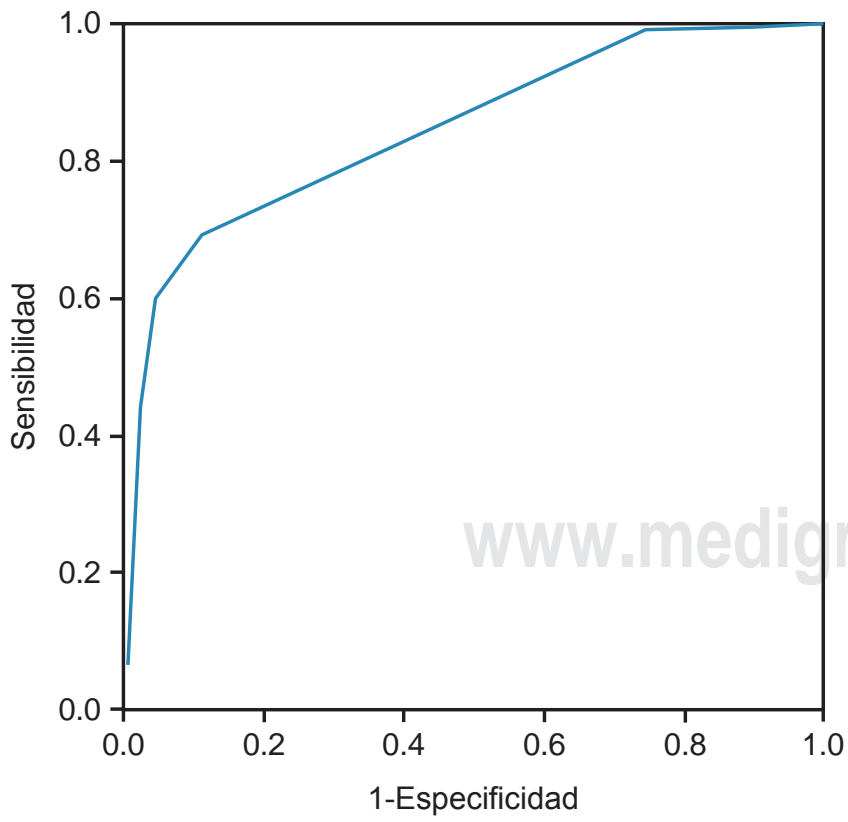

ROC BIRADS = Breast Imaging Report and Database System . biopsia. La tasa de falsos negativos es de $10-30 \% .{ }^{8-10}$ En nuestro estudio los casos con BIRADS 4B, 4C y 5 fueron 29,68 y $85 \%$ malignos, respectivamente. La proporción de falsos positivos BIRADS 5 en nuestro estudio fue de $15 \%$. Concluimos que esta diferencia se debe a que los estudios mamarios fueron realizados en diferentes gabinetes $y$, por lo tanto, interpretados por diferentes médicos radiólogos, lo que provoca un sesgo en la interpretación del estudio, ya sea consecuencia de la experiencia del médico o el sistema con el que se realiza el estudio.

Sin duda alguna, en un sector privado como el nuestro es difícil poder unificar el gabinete, ya que involucra otra variable importante para el paciente: el valor monetario. Sin embargo, esta variabilidad es tomada en cuenta para individualizar el protocolo de estudio de estos pacientes. Se realiza biopsia quirúrgica a aquellas pacientes clasificadas BIRADS 5 y con biopsia por VACORA benignas, que además presentan alta sospecha clínica de malignidad o de lo contrario, a aquéllas con sospecha clínica baja se les realiza seguimiento estrecho a uno, tres y seis meses; si durante este periodo presentan cambios importantes, se valora nueva biopsia por VACORA o biopsia quirúrgica.

La adición de la ecografía a la mastografía aumenta la sensibilidad para los cánceres pequeños, pero disminuye en gran medida la especificidad. En una revisión sistemática de estudios de cribado suplementario con ultrasonido en mujeres con una mastografía negativa, más de 90\% de los resultados positivos fueron falsos positivos. ${ }^{5} \mathrm{La}$ sensibilidad de la ecografía fue de 80 a $83 \%$, la especificidad de 86 a $94 \%$ y el valor predictivo positivo de 3 a $8 \%$; las tasas de retiro fueron de $14 \%$, en comparación con nuestro estudio en el que se obtuvieron valores con una sensibilidad de 0.99, una especificidad de 0.91, un valor predictivo positivo de 0.85 , y negativo de 0.98 , así como una exactitud de 0.42 (todos ellos a nivel global, siendo una prueba más precisa cuando se trató de casos BIRADS 4 c y 5 , cuando los valores se encontraron más 
altos en todos los rubros incluyendo exactitud de 0-91 y 0.93, respectivamente).

Las anormalidades en la mastografía incluyen: masas, calcificaciones, asimetría y distorsión de la arquitectura. El significado de estos hallazgos dependerá de la apariencia radiológica, así como de la edad de la paciente y la raza. El hallazgo más específico en la mastografía compatible con malignidad es una masa focal especulada, el cual tiene un valor predictivo de $81 \%$ en estudios a nivel mundial. ${ }^{8-10} \mathrm{Sin}$ embargo, el ultrasonido suplementario puede caracterizar, como benigna o maligna, todavía más una masa detectada mastográficamente o un área de distorsión arquitectónica. En un estudio en USA, la sensibilidad de los ultrasonidos mamarios por malignidad fue de $98.4 \%$ y el valor predictivo negativo de $99.5 \%{ }^{11}$

Se ha observado que identificar una masa quística por ultrasonido mamario no necesita más intervención porque el riesgo de cáncer es muy bajo. Una serie realizada por el U.S. Cancer Statistics Working Group no encontró tumores malignos en 223 quistes. ${ }^{12}$ La presencia de una masa intraquística debe provocar una aspiración con aguja fina (FNA) o una biopsia central de la masa con la intención de descartar una lesión mamaria maligna. En nuestro estudio pudimos observar que el hallazgo radiológico más común fue una masa ultrasonográfica en $87.9 \%$ de los casos, siendo la asociación de masa con calcificaciones la que estuvo más asociada con malignidad (sensibilidad de 0.76 o $76 \%$ ).

Recientemente ha ganado el centro de atención en diferentes estudios el concepto de "sobrediagnóstico" de cáncer de mama, que postula que algunos cánceres de mama detectados en un estudio de cribado nunca serán conocidos por el paciente o por su médico, mencionando que tales cánceres nunca producirán signos o síntomas clínicos y de la misma forma no representan una causa de muerte.

Cuando se usa como un suplemento a la mastografía, la ecografía puede mejorar la sensibilidad de la detección a expensas de una menor especificidad y una mayor tasa de biopsia de seno. En algunos estudios se ha podido observar que el uso de ultrasonido suplementario condujo al número de biopsias innecesarias a un aumento de cinco veces, en comparación con la mamografía sola. ${ }^{13}$ La tasa de biopsia positiva para las biopsias generadas por el cribado suplementario oscila entre 6 y $8 \%$, en comparación con 25 a $40 \%$ para las biopsias generadas por la mamografía de cribado sola. ${ }^{14}$ El sobrediagnóstico es completamente diferente de un "falso positivo en una biopsia", el cual es un hallazgo radiológico anómalo sospechoso de malignidad y que, posterior a la biopsia, muestra un resultado benigno. Si el diagnóstico excesivo realmente ocurre en el mundo real, las mujeres con cánceres sobrediagnosticados podrían recibir tratamientos "innecesarios" como la lumpectomía, mastectomía, quimioterapia y radioterapia. Además, estas mujeres experimentarían la ansiedad innecesaria de saber que tienen cáncer de mama y, por lo tanto, ello representaría un supuesto daño. Sin embargo, se ha reportado en varios metaanálisis y revisiones sistemáticas que el sobrediagnóstico de cáncer de mama es realmente bajo, con reportes menores a $5 \%$. Nuestro estudio no tiene como objetivo analizar el sobrediagnóstico de cáncer de mama, pero nuestros valores de sensibilidad y especificidad son muy altos, con lo que inferimos que el sobrediagnóstico se encuentra muy por debajo de $5 \%$, sin embargo, no descartamos la presencia de falsos positivos, además de que a todos los pacientes con sospecha radiológica alta se les realiza una estrecha vigilancia. Cabe mencionar que nosotros consideramos que los estragos del sobrediagnóstico tienen menos importancia clínica que los beneficios de la detección temprana.

\section{CONCLUSIONES}

Los valores de las pruebas diagnósticas obtenidas en el estudio son congruentes con los reportados a nivel mundial. A pesar del elevado porcentaje de falsos positivos (15\%) en estudios radiológicos y la probabilidad de sobrediagnóstico, concluimos que el uso complementario de USG mamario es de suma importancia, ya que aumenta los valores de sensibilidad para la detección de cáncer de mama.

\section{REFERENCIAS}

1. Lazcano E, Escudero P, Uscanga S. Cáncer de mama, diagnóstico, tratamiento, prevención y control. México: Ediciones ciencia; 2014.

2. Cárdenas SJ, Bargalló RJ, Bautista PV, Cervantes SG, Erazo VS, Fores $\mathrm{BC}$ et al. Consenso Mexicano sobrediagnóstico y tratamiento del cáncer mamario. Gaceta Mexicana de Oncología. 2015; 14 (Supl 2): 2-55.

3. Rodríguez CS, Guisa HF, Labastida AS, Espejo FR, Capurso GM, Ruvalcaba LE et al. Resultados del primer programa de detección oportuna de cáncer de mama en México mediante pesquisa con mastografía. GAMO. 2009; 8 (3): 83-96.

4. Masroor I, Rasool M, Afzal SS, Sohail S. To asses inter-and intraobserver variability for breast density and BIRADS assessment categories in mammographic reporting. J Pak Med Assoc. 2016; 66 (2): 194-197.

. Melnikow J, Fenton JJ, Whitlock EP, Miglioretti DS, Weyrich MS, Thompson JH, Shah K. Supplemental screening for breast cancer in women with dense breasts: a systematic review for the U.S. preventive service task force. Ann Intern Med. 2016; 164 (4): 268-278.

6. Slanetz PJ, Freer PE, Birdwell RL. Breast-density legislation--practical considerations. N Engl J Med. 2015; 372: 593-595.

7. Gartlehner G, Thaler K, Chapman A. Mammography in combination with breast ultrasonography versus mammography for breast cancer screening in women at average risk. Cochrane Database Syst Rev. 2013; 2013 (4): CD009632. 
8. Bland KI, Copeland EM, Gradishar WJ. The breast comprehensive management of benign and malignant diseases. 4a ed. Philadelphia, PA: Saunders Elsevier; 2009.

9. Medina PG, Paraguasú MG. Positive predictive value of Breast Imaging Reporting and Data System (BI-RADS ${ }^{\circledR}$ ) categories 3, 4 and 5. Radiol Bras. 2010; 43 (3): 171-174.

10. Kopans DB. The positive predictive value of mammography. AJR. 1992; 158: 521-526.

11. Boyd NF, Martin LJ, Yaffe MJ, Minkin S. Mammographic density and breast cancer risk: current understanding and future prospects. Breast Cancer Res. 2011; 13: 223.
12. U.S. Cancer Statistics Working Group. U.S. Cancer statistics data visualizations tool [sede web]. US: Center for Disease Control and prevention; 2019. Disponible en. https://www.cdc.gov/cancer/uscs/ pdf/uscs-data-visualizations-tool-technical-notes-h.pdf

13. Scheel JR, Lee JM, Sprague BL, Lee Cl, Lehman CD. Screening ultrasound as an adjunct to mammography in women with mammographically dense breasts. Am J Obstet Gynecol. 2015; 212: 9-17.

14. Brem RF, Lenihan MJ, Lieberman J, Torrente J. Screening breast ultrasound: past, present, and future. AJR Am J Roentgenol. 2015; 204: 234-240. 